\title{
Reexamining the Role of the Traumatic Stressor and the Trajectory of Posttraumatic Distress in the Wake of Disaster
}

\author{
Frederick C. Nucifora Jr, PhD, DO, MHS; Ryan C. Hall, MD; George S. Everly Jr, PhD
}

A

s we remember the 10th anniversary of the September 11, 2001 (9/11) terrorist attacks, Disaster Medicine and Public Health Preparedness is fortunate to publish 2 papers that address the long-term follow-up of first responders and citizens who were exposed to the events on that day, and a longitudinal study of survivors and first responders from the Hurricane Katrina disaster. These articles in many ways highlight why this journal was created: to allow for the study of disasters and aid in planning disaster response. These 3 papers provide the opportunity to reexamine not only the trajectory of posttraumatic distress in the wake of disaster but also the role of the stressor in that trajectory.

Soo et $\mathrm{al}^{1}$ review the effects of posttraumatic stress disorder (PTSD) on first responders to the terrorist attacks on the World Trade Center (WTC) towers. Their study (beginning October 2, 2001) focuses on firefighters who participated in the WTC rescue and recovery mission. They show that although most firefighters either did not develop probable PTSD (PPTSD) or they recovered from it, a portion of the firefighters continued to experience PPTSD 9 years after the terrorist events. Soo et al demonstrate that the firefighters who arrived on the scene first were more likely to develop and less likely to recover from PPTSD. The investigators also show that aerodigestive symptoms and a decrease in exercise resulting from any reason lower the likelihood of recovery. This research is significant because it demonstrates that responders who are first on the scene may experience more adverse mental health consequences, although we are unsure why this may be the case. Is there something uniquely toxic about being first on the scene? Does being first on the scene represent an important variable that can serve to intensify the adversity associated with a traumatic stressor? If so, how can we act to buffer this unique psychological toxicity? This article is also significant because it offers the longest-term perspective on this first-responder population after the 9/11 attacks.

In the second article, Osofsky et $\mathrm{al}^{2}$ examine the mental health of first responders to Hurricane Katrina to determine the rates of PTSD and depression. They assess first responders at 6 to 9 months and then reassess a portion of those individuals at 13 to 18 months. They show that there is a percentage of responders that meet PPTSD and depression criteria and that the rates do not change significantly between time points. They also show that there was an increase in alcohol consumption and partner conflict that appears stable at both assessment points. The significance of this article is that it highlights, as does the study of Soo et al, the vulnerable population that is first responders. It also raises questions as to why there was no decline in PPTSD as one may predict would occur naturally. Clearly, the qualitative nature of the WTC disaster is different from that of Hurricane Katrina, especially in New Orleans. Can these differences serve to inform enhanced mental health-response capabilities?

The third paper, by North et $\mathrm{al}^{3}{ }^{3}$ considers the rates of PTSD based on subjects' geographical distance from the WTC towers during the attacks. The subjects include employees of 8 organizations in New York City with a range of exposures, including evacuees from the WTC towers and others in the adjacent vicinity. Their data demonstrate that geographical distance did not predict PTSD symptom criteria beyond a short distance from the towers. This article is significant because the authors performed a diagnostic interview and based their diagnosis of PTSD on the full Diagnostic and Statistical Manual, Fourth Edition, Text Revision criteria. They specifically examine qualifying exposures, and discuss the advantages of including such an inquiry.

The 3 articles provide an opportunity to reexamine the trajectory of posttraumatic distress, as well as the variable of stressor exposure itself, in the wake of disasters. In addition, they point to questions that may be addressed by future research. North et al raise the issue of the importance of exposure criteria and the possibility that PTSD may be overdiagnosed in certain populations and studies. Osofsky et al and Soo et al use validated survey items and checklists to diagnose PPTSD; however, because the subjects did not undergo a psychiatric interview, it cannot be said for certain that they meet the requirements for PTSD. North et al addressed this issue by using diagnostic interviews. It is interesting to note that they show that qualifying exposure for PTSD often requires close geographical proximity to the incident and that $80 \%$ of the people who had PTSD symptoms but did not meet exposure criteria had received another psychiatric diagnosis. There is a precedent, some may argue, for the reporting of exaggerated figures of PTSD when exposure and an adequate history are not taken or confirmed. For example, The National Vietnam Veterans Readjustment Study stated that almost 1 million soldiers (830 000) who served in the Vietnam theater experienced PTSD, but later 
investigations raised doubts about this number when it was found that only about 300000 of these individuals were actually assigned to combat units. ${ }^{4-7}$

Accurately diagnosing PTSD is complicated further by the problem that many of the symptoms of PTSD overlap with other psychiatric illnesses and that $44 \%$ to $88 \%$ of individuals with PTSD present with at least 1 other psychiatric condition. $^{8-10}$ These findings lead to disagreement in the literature about whether PTSD imitates other conditions or whether it is multiple conditions occurring independently. In addition, longitudinal studies may misinterpret de novo mental health conditions as being directly related to the disaster. Just because there is a correlation between exposure and symptoms does not mean that someone developed symptoms as a result of that specific event. For example, it is unclear from Soo et al whether any of the firefighters had other exposures either before or after $9 / 11$. It is likely that a majority of these firefighters were exposed to other traumas because of the nature of being a first responder. ${ }^{11}$ They also may have been undergoing personal traumas and stress unrelated to work. The symptoms reported on a research checklist may have been better attributed to other causes or disease states, even though the subjects were being asked about $9 / 11$, because the exact cause for symptoms may be hard for the individual experiencing them to determine.

It is important that people be assessed properly in the clinical setting and in disaster studies for several reasons. First, assessment should guide treatment. Someone with depression may need a different treatment than someone who has alcoholism, psychotic break, personality decompensation, or PTSD. Diagnosing individuals as having PTSD who do not meet qualifying exposures could help explain some of the failure rates of in vivo desensitization, and in this case, it has the potential of not only failing to adequately help individuals but also it may actually do harm. With regard to adverse iatrogenesis, recently published research by Boscarino et $\mathrm{al}^{12}$ using a well-controlled longitudinal design, found that the best predictor of mental health service utilization postdisaster was higher exposure. Perhaps more important, their research suggests that brief mental health interventions (crisis intervention) appeared to be more effective than traditional multisession therapy. Furthermore, traditional therapy actually was associated with a worsening of symptoms. Second, to inform best practices for the treatment of psychiatric illnesses, it is critical for researchers to have a "clean" population to study to determine the pathophenomenology of these illnesses. Third, misattributing the prevalence of a disorder could inadvertently shift already limited resources away from where they are most needed. To facilitate accurate diagnosis, and thus improve intervention, individuals must be studied over time using diagnostic interviews, and each subject must be considered from multiple perspectives, including a person's behaviors, life stories, person- ality vulnerabilities and any prior or unrelated de novo mental disorders.

Not only does each article describe the long-term population effects of disaster and the importance of the dynamics of stressor exposure, but also we believe that they support the notion that a continuum of care model is needed when formulating a disaster mental health plan as part of disaster preparedness. As we have described in this journal and elsewhere, ${ }^{13-15}$ the model of resistance, resilience, and recovery (RRR) is a longitudinal model that holds promise in helping disaster survivors and first responders prepare for disaster and cope with the aftereffects. The RRR model has several advantages over traditional and noncontinuum of care models. In general, traditional models are limiting and focus only on some aspects of behavior. They tend to be inflexible and event focused, they do not include assessment of individuals or organizations, and they do not make hypotheses that can be assessed postevent to establish effectiveness. They also tend to be reactionary, to not be proactive, and to propose one-size-fits-all interventions, ignoring vulnerabilities. The RRR model is an integrative proactive approach that helps address the needs of individuals and groups at each stage of the process and allows for hypothesis testing. Furthermore, Schoenbaum et $\mathrm{al}^{16}$ have shown that implementing variations on the theme of a continuum of care can reduce the cost of subsequent mental health utilization.

The first component of the RRR model is resistance. Resistance refers to the ability of an individual, a group, an organization, or even an entire population to withstand manifestations of clinical distress, impairment, or dysfunction associated with critical incidents, terrorism, and even mass disasters. Resistance may be thought of as a form of psychological "immunity" to distress and dysfunction, analogous to preillness vaccination. Resistance training, much like vaccinations, may also result in a "herd immunity," in which inoculated individuals help prevent the spread of fear, panic, and confusion to others. Resistance is an important concept and distinct from resilience because it is a proactive preincident intervention and suggests that people can be immunized against the perturbations of disasters and therefore experience little to no significant dysfunction after an event. Effective resistance training is ideal for the populations discussed in the articles by Soo et al and Osofsky et al. By understanding what constitutes resistance we may be able to inoculate first responders from many traumatic events, with the possibility of eventually immunizing the community at large.

Although there is a great need for studies on how to build resistance, we do know some general basics derived from social learning theory and the construct of self-efficacy. Selfefficacy, a belief in personal agency, appears to affect how people respond to stress. "People's beliefs in their efficacy ... influ- 
ence the courses of action people choose to pursue, how much effort they put forth in given endeavors, how long they will persevere in the face of obstacles and failures, their resilience to adversity, whether their thought patterns are self-hindering or self-aiding, how much stress and depression they experience in coping with taxing environmental demands, and the level of accomplishments they realize." 17 People with high selfefficacy tend to have a strong sense of purpose. Self-efficacy can be built by direct experience, vicarious experiences, verbal support, education, and managing physiologic and affective arousal. Self-efficacy also may be enhanced by leadership. ${ }^{8,18}$

The immediate postevent phase of the RRR model is resilience, which refers to the ability of an individual, a group, an organization, or even an entire population to rapidly and effectively rebound from psychological perturbations associated with critical incidents, terrorism, and even mass disasters. As typified by Boscarino et $\mathrm{al}^{12}$ and dating back to studies on community psychiatry ${ }^{19}$ and combat psychiatry, ${ }^{20,21}$ there is evidence that resilience can be enhanced through crisis intervention and psychological first-aid practices. Resilient leadership practices may be of assistance as may effective risk communication, fostered positive cognitions, information and rumor deterrence, reassurance, direction, motivation and a sense of connectedness. ${ }^{8,11,18,22}$ This may be particularly important for first responders and survivors closest to the event because both North et al and Soo et al suggest that they suffer the most after traumatic events. There is evidence in front-line treatment of combat stress reactions that providing interventions close to the event in proximity in time and having an expectation that people can recover rapidly and resume functioning can lower rates of psychiatric symptoms, decrease loneliness, and lead to better social functioning. ${ }^{21}$ All of these concepts can be applied to any type of disaster to help individuals rebound.

The final part of the model is recovery, which refers to the ability of an individual, a group, an organization, or even an entire population literally to recover their adaptability and function, both psychologically and behaviorally, in the wake of significant clinical distress, impairment, or dysfunction subsequent to critical incidents such as terrorism, acts of violence, and even mass disasters. Recovery will be needed for the percentage of people who still have perturbations years after the disaster and involves psychiatric treatment. The 3 articles in this issue of the journal identify a population of individuals who are in need of additional recovery and would be best served by a full psychiatric evaluation and treatment.

These articles identify a population that continues to suffer long after a disaster, regardless of the diagnosis, and directly or indirectly raises the question of how best to apply research and improve design for future study and resource allocation. It is time to move past collecting statistics on how many people develop psychiatric consequences after a disaster and start engaging in more prospective study design. Although these may be difficult studies for many reasons (eg, we cannot predict where or when a disaster will strike), we have the opportunity to study a defined and easily accessed population in first responders as was evident from the studies in this issue. As a field, disaster medicine needs to examine preincident training and develop programs to build resistance that take into account antecedent factors such as baseline psychiatric functioning, history, and personality features. ${ }^{8-10,22} \mathrm{We}$ can design trials to increase self-efficacy and determine which ones build resistance and enhance resilience. We can design prospective studies to address ways to prepare people for disasters and determine which have the best outcomes. To better understand PTSD, we should strive to design studies that identify cohorts of people who meet PTSD criteria without comorbidities. Careful and accurate diagnosis will aid neuroscientists in their quest to determine the pathophysiology behind these illnesses, with the goal of developing better treatments and perhaps even prevention strategies. Accurate diagnosis will provide state and local governments with the proper information when allocating scarce resources and trying to predict long-term outcomes. The goal of this type of research is to find a better way to provide help and support to our everyday heroes.

Author Affiliations: Drs Nucifora and Everly are with the Department of Psychiatry and Behavioral Sciences, Johns Hopkins University School of Medicine; Dr Hall is with the Department of Psychiatry, University of South Florida.

Correspondence: Address correspondence and reprint requests to Dr Frederick C. Nucifora Jr, Department of Psychiatry, Johns Hopkins University School of Medicine, $600 \mathrm{~N}$ Wolfe St, CMSC 8-121, Baltimore, MD 21287 (e-mail: nucifora@jhmi.edu).

Received for publication July 11, 2011; accepted July 13, 2011.

Author Disclosures: The authors report no conflicts of interest.

\section{REFERENCES}

1. Soo J, Webber MP, Gustave J, et al. Trends in probable PTSD in firefighters exposed to the World Trade Center disaster, 2001-2010. Disaster Med Public Health Prep. 2011;5(Suppl 2):S197-S203.

2. Osofsky HJ, Osofsky JD, Arey J , et al. Hurricane Katrina's first responders: the struggle to protect and serve in the aftermath of the disaster. Disaster Med Public Health Prep. 2011;5(Suppl 2):S214-S219.

3. North CS, Pollio DE, Smith RP, et al. Trauma exposure and posttraumatic stress disorder among employees of New York City companies affected by the September 11, 2001 attacks on the World Trade Center. Disaster Med Public Health Prep. 2011;5(Suppl 2):S205-S213.

4. Price JL. Findings from the National Vietnam Veterans' Readjustment Study. http://www.ptsd.va.gov/professional/pages/vietnam-vets-study.asp. Published January 1, 2007. Accessed July 8, 2011.

5. Shephard B. A War of Nerves: Soldiers and Psychiatrists 1914-1994. London: Johnathan Cape; 2000:392.

6. McHugh PR, Treisman G. PTSD: a problematic diagnostic category. J Anxiety Disord. 2007;21(2):211-222.

7. McNally RJ. Can we solve the mysteries of the National Vietnam Veterans Readjustment Study? J Anxiety Disord. 2007;21(2):192-200.

8. Hall RC, Hall RC, Chapman MJ. Effects of terrorist attacks on the elderly: part II. Posttraumatic stress, acute stress, and affective disorders. Clin Geriatr. 2006;14:17-24.

9. Hall RC, Hall RC. Malingering of PTSD: forensic and diagnostic consid- 
erations, characteristics of malingerers and clinical presentations. Gen Hosp Psychiatry. 2006;28(6):525-535.

10. Hall RC, Hall RC. Detection of malingered PTSD: an overview of clinical, psychometric, and physiological assessment: where do we stand? J Forensic Sci. 2007;52(3):717-725.

11. Hall RC, Hall RC, Chapman MJ. Emotional and psychiatric effects of weapons of mass destruction in first responders. In: Ursano RJ, Norwood AE, Fullerton CS, eds. Bioterrorism: Psychological and Public Health Interventions. Cambridge, UK: Cambridge University Press; 2004:250-273.

12. Boscarino JA, Adams RE, Figley CR. Mental health service use after the World Trade Center disaster: utilization trends and comparative effectiveness. J Nerv Ment Dis. 2011;199(2):91-99.

13. Kaminsky MJ, McCabe OL, Langlieb A, Everly GS Jr. An evidenceinformed model of human resistance, resilience, \& recovery: the Johns Hopkins' outcomes-driven paradigm for disaster mental health services. Brief Ther Crisis Intervention. 2006;7:1-11.

14. Nucifora F Jr, Langlieb AM, Siegal E, Everly GS Jr, Kaminsky MJ. Building resistance, resilience, and recovery in the wake of school and workplace violence. Disaster Med Public Health Prep. 2007;1(1)(Suppl):S33S37.
15. Everly GS Jr. Fostering Human Resilience in Crisis. Ellicott City, MD: Chevron Publishing; 2011.

16. Schoenbaum M, Butler B, Kataoka S, et al. Promoting mental health recovery after hurricanes Katrina and Rita: what can be done at what cost. Arch Gen Psychiatry. 2009;66(8):906-914.

17. Bandura A. Self-Efficacy: The Exercise of Control. New York: Freeman; 1997.

18. Everly GS Jr, Strouse DA, Everly GS III. Secrets of Resilient Leadership. New York: DiaMedica; 2010.

19. Decker JB, Stubblebine JM. Crisis intervention and prevention of psychiatric disability: a follow-up study. Am J Psychiatry. 1972;129(6):725729.

20. Solomon Z, Benbenishty R. The role of proximity, immediacy, and expectancy in frontline treatment of combat stress reaction among Israelis in the Lebanon War. Am J Psychiatry. 1986;143(5):613-617.

21. Solomon Z, Shklar R, Mikulincer M. Frontline treatment of combat stress reaction: a 20-year longitudinal evaluation study. Am J Psychiatry. 2005; $162(12): 2309-2314$

22. Hall RC, Hall RC, Chapman MJ. Medical and psychiatric casualties caused by conventional and radiological (dirty) bombs. Gen Hosp Psychiatry. 2006;28(3):242-248. 